\title{
Dynistor Switches for Micro- and Nanosecond Power Pulse Generators
}

\author{
Yu.V. Aristov, I.V. Grekhov, S.V. Korotkov and A.G. Lyublinsky* \\ Ioffe Physico-Technical Institute, Russian Academy of Sciences \\ 26 Polytechnicheskaya, St. Petersburg, 194021 Russia
}

\begin{abstract}
Gigawatt power switches for microsecond range applications based on an assembly of Reverse Switch-on Dynistors (RSD) and fast compact generators for nanosecond range applications based on new semiconductor devices Deep Level Dynistors (DLD) are discussed. As an example design and operation principles of $25 \mathrm{kV}$, $300 \mathrm{kA}, 600 \mu \mathrm{s}$ pulse duration power switch based on $76 \mathrm{~mm}$ diameter RSDs and $25 \mathrm{kV}, 10 \mathrm{kA}$ switch based on $16 \mathrm{~mm}$ diameter DLDs are described. It is possible to increase the pulse power by increasing both diameter of dynistor structure and number of devices connected in series or in parallel.
\end{abstract}

PACS numbers: 84.70.+p, 84.30.Ng, 85.30.-z, 85.30.De

\section{Introduction}

At present the most powerful wide used semiconductor switches are pulse power thyristors for microsecond range applications and MOS-controlled thyristors (SMCT) for nanosecond applications.

Two electrodes semiconductor device Reverse Switched-on Dynistor (RSD) [1, 2] and new semiconductor device Deep Level Dynistor (DLD) $[3,4]$, which are perspective replacement for both these thyristors, have been developed in Ioffe Physical Technical Institute.

\section{Main operation principles}

Dense grid of reverse conductivity channels is introduced into RSD silicon structure to control the device. Reverse control current passes through the grid of channels while short pulse (less than $1 \mu \mathrm{s}$ ) of reverse voltage is applied to the dynistor. Power circuit is blocked during this time by saturable core choke. The control current causes electron-hole injection into the base regions of dynistor and uniform accumulation of initiating charge near the $p-N$ junction. When direct voltage is applied to dynistor, RSD is triggered and switching on process expands uniformly and simultaneously over the entire device area, which determines unique RSD switching on capabilities.

For example, RSD with $76 \mathrm{~mm}$ diameter of semiconductor structure commutates $300 \mathrm{kA}$ peak current with

* corresponding author; e-mail:

Alexander.Lyublinsky@mail.ioffe.ru up to $30 \mathrm{kA} / \mu$ s current rise rate, which considerably exceeds the commutating capabilities of pulse thyristors with the same diameter. It is important to note also that simple processing of RSD allows to obtain lower price of RSD in comparison with the price of the same size pulse thyristors.

RSD is microsecond range applications device. Modulation of RSD base regions by electron-hole plasma during switching on process occurs relatively slowly (few tenths of microsecond) because it is produced by bipolar drift of carriers injected by emitters. Therefore energy losses in RSD structure increase sharply if it operates at nanosecond pulse durations.

The energy losses for nanosecond pulses are considerably lower in DLD structure. DLD switches on by impact ionization front propagation which is formed by applying short (1-2 ns) overvoltage pulse with rise rate not less than $1 \mathrm{kV} / \mathrm{ns}$. DLD is a four layers two electrodes device on the base of silicon with deep levels at the middle of the band gap. When electric field exceeds static critical breakdown field in few times, the tunnel ionization of impurity centers occurs, electrons appear in the super high field region and cause the intensive impact ionization. The front of impact ionization moves with velocity 3-5 times higher than saturated velocity, leaving electron-hole plasma behind itself. This plasma with a high conductivity fills the base layers and causes fast DLD switching into conductive state. Typical duration of this process does not exceed several hundreds of picoseconds.

For example, DLD with $12 \mathrm{~mm}$ diameter of semiconductor structure and operating voltage $2.5 \mathrm{kV}$ commutates $10 \mathrm{kA}$ peak current with up to $250 \mathrm{~A} / \mathrm{ns}$ current rise rate, which considerably exceeds the commutating capabilities of SMCT thyristors. Cost of DLD is significantly lower than SMCT due to simple DLD technology. 


\section{Circuit design of RSD and DLD based generators}

The basic principal electric circuits of high voltage DLD and RSD based generators with capacitor energy storage $\mathrm{C}_{0}$ are shown in Figs. 1 and 2.

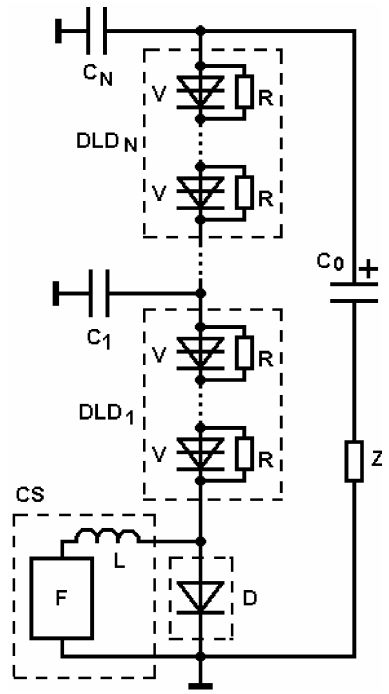

Fig. 1. Principal electrical circuit of DLD based generator.

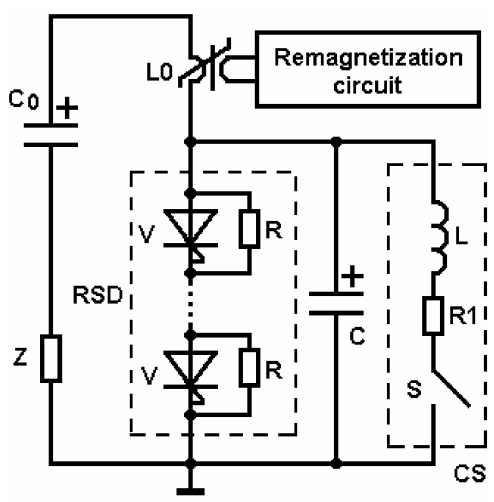

Fig. 2. Principal electrical circuit of RSD based generator.

$\mathrm{DLD}_{1}-\mathrm{DLD}_{N}$ stacks in Fig. 1 are triggered by control system CS, starting capacitors $\mathrm{C}_{1}-\mathrm{C}_{N}$ and diode current breaker $\mathrm{D}$ which is made of serially connected drift step recovery diodes (DSRD) [5]. Pulse former $\mathrm{F}$ provides required operation regime for DSRD.

At first short pulse $(\sim 100 \mathrm{~ns})$ of direct current passes through DSRD, electron and hole charges are stored into DSRD base layer in vicinity of injectors. After that reverse pulse current with about the same duration pulls out stored charge and provides DSRD switching off. The duration of current breaking by DSRD is no more that few nanoseconds at optimal reverse current density
(100-300 A/ $\left.\mathrm{cm}^{2}\right)$. DSRD switches off and current of inductor $\mathrm{L}$ is commutated into $\mathrm{C}_{1}-\mathrm{DLD}_{1}$ circuit. High voltage pulse occurs on $\mathrm{DLD}_{1}$ dynistors assembly and it switches on. Then voltage on $\mathrm{DLD}_{N}$ assembly sharply increases and it switches on, too. Thereby, $\mathrm{DLD}_{1}-\mathrm{DLD}_{N}$ assemblies are switched on one after another and energy storage capacitor $\mathrm{C}_{0}$ discharges through the load $\mathrm{Z}$.

Principal electrical circuit of RSD based generator is shown in Fig. 2. RSD stack is triggered by starting capacitor $\mathrm{C}$ and control system CS based on switch $\mathrm{S}$ which is usually made of DLD assembly.

At first switch $\mathrm{S}$ closes and discharge current of capacitor $\mathrm{C}$ passes through $\mathrm{LR}_{1}$ circuit. When the voltage on capacitor $\mathrm{C}$ changes to negative this reverse voltage applies to RSD stack and RSD control current formed by $\mathrm{L}$ inductor passes through dynistors structure. While the control current passes through RSD, saturable core choke $L_{0}$ has large inductance and prevents sharp increasing of power current through the load. When the choke of $L_{0}$ inductor saturates, direct voltage is applied to RSD, they switch on simultaneously and capacitor $\mathrm{C}_{0}$ discharges through the load.

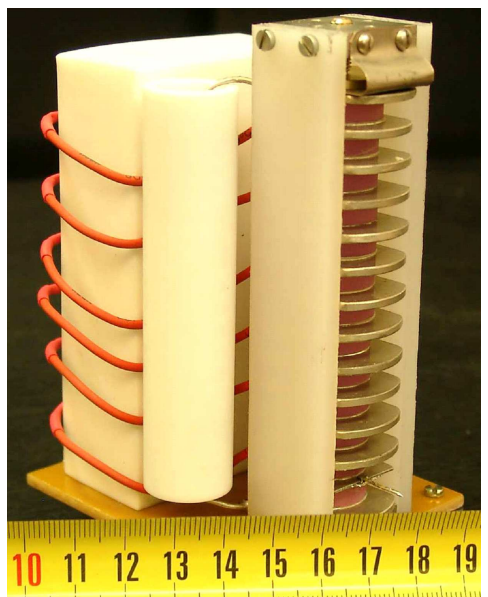

Fig. 3. $25 \mathrm{kV}$ nanosecond DLD based switch with $10 \mathrm{kA}$ peak current and $250 \mathrm{~A} / \mathrm{ns}$ current rise rate.

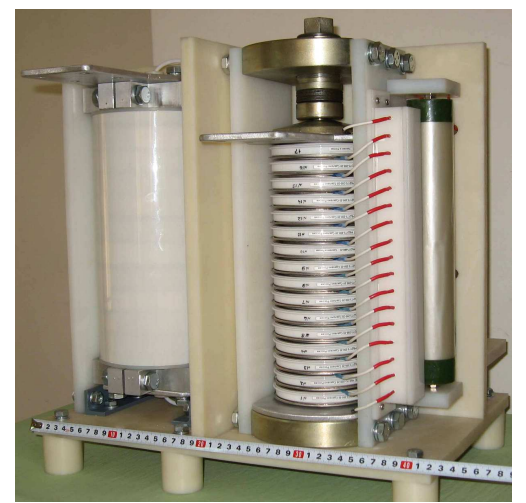

Fig. 4. $25 \mathrm{kV}$ microsecond RSD based switch with $300 \mathrm{kA}$ peak current and $30 \mathrm{kA} / \mu$ s current rise rate. 
The photos of designed DLD and RSD based switches with $25 \mathrm{kV}$ operating voltage are presented in Figs. 3 and 4. Nanosecond DLD based switch (Fig. 3) commutates current pulses with $10 \mathrm{kA}$ amplitude and up to $250 \mathrm{~A} / \mathrm{ns}$ current rise rate. RSD based switch (Fig. 4) commutates microsecond pulses with $300 \mathrm{kA}$ peak current and up to $30 \mathrm{kA} / \mu$ s current rise rate.

\section{Conclusions}

Nanosecond and microsecond range power generators and switches based on new semiconductor devices Reverse Switched-on Dynistor (RSD) and Deep Level Dynistor (DLD) were developed. The switching capability of RSD and DLD is significantly higher than those of the best modern pulse power thyristors and MOS-controlled thyristors correspondingly.

The possible application area of such dynistor switches and generators are lasers, acceleration and radar tech- niques, nuclear research, purification of industrial gases and water by electrical discharge, pulsed electromagnetic and electro hydraulic technologies etc.

\section{References}

[1] I.V. Grekhov, A.V. Gorbatyuk, L.S. Kostina, S.V. Korotkov, N.S. Iakovtchuk, Solid State Electron. 26, 1132 (1983).

[2] Yu.V. Aristov, V.B. Voronkov, I.V. Grekhov, A.L. Zhmodikov, A.K. Kozlov, S.V. Korotkov, I.A. Rol'nik, Instrum. Exp. Techniques 50, 228 (2007).

[3] I.V. Grekhov, S.V. Korotkov, P.B. Rodin, IEEE Trans. Plasma Sci. 36, 378 (2008).

[4] Yu.V. Aristov, V.B. Voronkov, I.V. Grekhov, A.K. Kozlov, S.V. Korotkov, A.G. Lyublinskii, Instrum. Exp. Techniques 50, 224 (2007).

[5] I.V. Grekhov, V.M. Efanov, A.F. Kardo-Susoev, S.V. Shenderey, Solid State Electron. 28, 597 (1985). 